Proyecciones Journal of Mathematics

Vol. 35, $\mathrm{N}^{o}$ 3, pp. 301-316, September 2016.

Universidad Católica del Norte

Antofagasta - Chile

\title{
Non-linear maps preserving singular algebraic operators
}

\author{
Mourad Oudghiri \\ Université Mohammed Premier - Oujda, Maroc \\ and \\ Khalid Souilah \\ Uiversité Mohammed Premier - Oujda, Maroc \\ Received: March 2016. Accepted : June 2016
}

\begin{abstract}
Let $\mathcal{B}(\mathcal{H})$ be the algebra of all bounded linear operators on an infinite-dimensional Hilbert space $H$. We prove that if $\Phi$ is a surjective map on $\mathcal{B}(\mathcal{H})$ such that $\Phi(I)=I+\Phi(0)$ and for every pair $T, S \in \mathcal{B}(\mathcal{H})$, the operator $T-S$ is singular algebraic if and only if $\Phi(T)-\Phi(S)$ is singular algebraic, then $\Phi$ is either of the form $\Phi(T)=A T A^{-1}+\Phi(0)$ or the form $\Phi(T)=A T^{*} A^{-1}+\Phi(0)$ where $A: H \rightarrow H$ is an invertible bounded linear, or conjugate linear, operator.
\end{abstract}

Subjclass [2000] : 47B49, 47L99, 47A55, 47B37.

Keywords : Non-linear preserver problems, Algebraic operators. 


\section{Introduction}

Throughout this paper, $X$ denotes an infinite-dimensional Banach space over the field $\mathbf{K}=\mathbf{R}$ or $\mathbf{C}$, and $\mathcal{B}(X)$ the algebra of all bounded linear operators on $X$.

Two operators are said to be adjacent if their difference is of rank one. A map $\Phi$ on $\mathcal{B}(X)$ onto itself is said to preserve adjacency in both directions if for every pair $A, B$ we have that $A$ and $B$ are adjacent if and only if $\Phi(A)$ and $\Phi(B)$ are adjacent.

Let $\mathcal{M}_{n}(\mathbf{K})$ be the space of all $n \times n$ matrices over $\mathbf{K}, n \geq 2$. The fundamental Hua's theorems of the geometry of matrices characterize the general form of bijective maps on various spaces of matrices (rectangular matrices, symmetric matrices, Hermitian matrices, etc.) which preserve adjacency in both directions. Specially, the well-known Hua's theorem from [7] states that a bijective map $\Phi$ on $\mathcal{M}_{n}(\mathbf{K})$ preserves adjacency in both directions if and only if $\Phi$ has one of the following two forms

$$
\left[a_{i j}\right] \mapsto P\left[\tau\left(a_{i j}\right)\right] Q+R \quad \text { or } \quad\left[a_{i j}\right] \mapsto P\left[\tau\left(a_{i j}\right)\right]^{\operatorname{tr}} Q+R
$$

where $P, Q, R \in \mathcal{M}_{n}(\mathbf{K})$ with $P, Q$ invertible, $A^{\text {tr }}$ denotes the transpose of $A$, and $\tau$ is an automorphism of $\mathbf{K}$. This beautiful theorem has many applications, for example in the theory of Jordan automorphisms and Lie automorphisms. For other applications of results on adjacency preserving maps, particularly in the theory of local homomorphisms, linear preserver problems, and graph theory, we refer to [5, 14] and to Wan's book [19], where most of the known results on the geometry of matrices are collected.

Some recent improvements of this theorem can be found in $[8,15,16]$. In [14], Šemrl has extended the fundamental Hua's theorem of the geometry of rectangular matrices to the infinite-dimensional case.

Let $\Lambda$ be a subset of $\mathcal{B}(X)$. We shall say that a map $\Phi: \mathcal{B}(X) \rightarrow \mathcal{B}(X)$ preserves the difference of $\Lambda$ in both directions if for every $T, S \in \mathcal{B}(X)$,

$$
T-S \in \Lambda \text { if and only if } \Phi(T)-\Phi(S) \in \Lambda \text {. }
$$

Recently, Havlicek and Šemrl have shown in [5] that a bijective map $\Phi$ on $\mathcal{B}(\mathcal{H})$, where $H$ is an infinite-dimensional complex Hilbert space, preserves the difference of invertible operators in both directions if and only if it has one of the following two forms

$$
T \mapsto A T B+R \quad \text { or } \quad T \mapsto A T^{*} B+R,
$$


where $A, B: H \rightarrow H$ are simultaneously invertible bounded linear or conjugate linear operators, $R \in \mathcal{B}(\mathcal{H})$, and $T^{*}$ denotes the Hilbert space adjoint of $T$. This result is extended in [6] to the setting of infinite-dimensional complex Banach spaces. Later, in [3] the authors established a similar result of [6] by considering injective operators, surjective operators, and bounded below operators.

The problems of the characterization of maps $\Phi$ on $\mathcal{B}(X)$ preserving the difference of $\Lambda$ in both directions can be considered as generalizations of linear preservers problems of $\Lambda$ which concern the characterization of linear, or additive, maps $\Psi$ on $\mathcal{B}(X)$ that preserve $\Lambda$ in both directions, that is, for every $T \in \mathcal{B}(X)$,

$$
T \in \Lambda \text { if and only if } \Psi(T) \in \Lambda \text {. }
$$

For excellent expositions on linear preserver problems, we refer the reader to $[1,2,9,17]$ and the references therein.

Let $\mathcal{B}_{0}(X)$ denote the linear span of all nilpotent operators in $\mathcal{B}(X)$. In $[2]$, the authors showed that a surjective additive map $\Phi: \mathcal{B}_{0}(X) \rightarrow \mathcal{B}_{0}(X)$ preserves nilpotent operators in both directions if and only if it has one of the following two forms

$$
T \mapsto c A T A^{-1} \quad \text { or } \quad T \mapsto c A T^{\prime} A^{-1}
$$

where $c$ is a non-zero scalar, $A$ is a bounded invertible linear, or conjugate linear, operator between suitable spaces and $T^{\prime}$ denotes the adjoint of $T$ on the topological dual space $X^{\prime}$ of $X$. In the special case of a Hilbert space $H$, this result is extended to linear maps defined on the whole space $\mathcal{B}(\mathcal{H})$. The same authors obtained in [1] a complete description of surjective linear maps on $\mathcal{B}(\mathcal{H})$ preserving operators annihilated by a given complex polynomial $P$ with $\operatorname{deg}(P) \geq 2$ in both directions. Thus, they extended several results on linear mappings preserving square zero operators, idempotent operators or $r$-potent operators $T$; that satisfy $T^{r}=T$.

Clearly, every map $\Phi$ on $\mathcal{B}(X)$ having one of the forms (1.1) preserves the difference of nilpotent operators in both directions, and if $c=1$ it preserves also the difference of idempotent operators in both directions. The converse is generally false as can be shown by considering the map $\Psi(T)=T+I$ for all $T \in \mathcal{B}(X)$. It seems then interesting to ask what can we say about non-linear maps preserving the difference of nilpotent operators, idempotent operators or, more generally, algebraic operators in both directions? This problem is still open. However, in the present paper, we give a partial answer. 
Recall that an operator $T \in \mathcal{B}(X)$ is singular algebraic if $T$ is noninvertible and there exists a non-zero complex polynomial $P$ such that $P(T)=0$. Let $\mathcal{A}_{s}(X)$ denote the set of all singular algebraic operators in $\mathcal{B}(X)$, and $\mathcal{A}_{0}(X)$ the linear span of all algebraic operators in $\mathcal{B}(X)$. It is worth mentioning that $\mathcal{A}_{s}(X)$ contains rank one operators, nilpotent operators and idempotent operators except $I$.

Our purpose is to describe the general structure of surjective mappings $\Phi$ on $\mathcal{A}_{0}(X)$ preserving the difference of singular algebraic operators in both directions. More precisely, the main result of this paper can be summarized in the following theorem :

Theorem A Let $\Phi: \mathcal{A}_{0}(X) \rightarrow \mathcal{A}_{0}(X)$ be a surjective map such that $\Phi(I)=I+\Phi(0)$. Then the following assertions are equivalent :

(i) $\Phi$ preserves the difference of $\mathcal{A}_{s}(X)$ in both directions;

(ii) there exists either a bounded invertible linear, or conjugate linear, operator $A: X \rightarrow X$ such that

$$
\Phi(T)=A T A^{-1}+\Phi(0) \quad \text { for all } T \in \mathcal{A}_{0}(X),
$$

or, a bounded invertible linear, or conjugate linear, operator $B: X^{\prime} \rightarrow$ $X$ such that

$$
\Phi(T)=B T^{\prime} B^{-1}+\Phi(0) \quad \text { for all } T \in \mathcal{A}_{0}(X) .
$$

Let $H$ be an infinite-dimensional Hilbert space. For $T \in \mathcal{B}(\mathcal{H})$, note that $T^{*}=\mathrm{J}_{\mathrm{o}}^{-1} T^{\prime} \mathrm{J}_{\mathrm{o}}$ where $\mathrm{J}_{\mathrm{o}}: H \rightarrow H^{\prime}$ is the natural conjugate linear mapping defined by

$$
\mathrm{J}_{\mathrm{o}}(x)(u)=<u, x>\quad \text { for all } x, u \in H .
$$

By a result of Pearcy and Topping [13], every operator in $\mathcal{B}(\mathcal{H})$ is a finite sum of square-zero operators, which implies that $\mathcal{A}_{0}(H)=\mathcal{B}(\mathcal{H})$. Hence, we obtain the following theorem as an immediate consequence of the previous one.

Theorem B Let $H$ be an infinite-dimensional Hilbert space, and let $\Phi$ : $\mathcal{B}(\mathcal{H}) \rightarrow \mathcal{B}(\mathcal{H})$ be a surjective map such that $\Phi(I)=I+\Phi(0)$. The following assertions are equivalent : 
(i) $\Phi$ preserves the difference of $\mathcal{A}_{s}(H)$ in both directions;

(ii) there exists an invertible bounded linear, or conjugate linear, operator $A$ on $H$ such that either

$$
\Phi(T)=A T A^{-1}+\Phi(0) \quad \text { for all } T \in \mathcal{B}(\mathcal{H})
$$

or

$$
\Phi(T)=A T^{*} A^{-1}+\Phi(0) \quad \text { for all } T \in \mathcal{B}(\mathcal{H})
$$

The paper is organized as follows. The second section is devoted to the characterization of the adjacency of operators in term of singular algebraic operators. In the last section, we will prove our main theorem.

\section{Singular algebraic operators and adjacency}

For an operator $T \in \mathcal{B}(X)$, write $\operatorname{Ker}(T)$ for its kernel, $\operatorname{ran}(T)$ for its range, and $\sigma(T)$ for its spectrum. Let $z \in X$ and $f \in X^{\prime}$ be non-zero. We denote, as customary, by $z \otimes f$ the rank one operator given by $(z \otimes f)(x)=f(x) z$ for all $x \in X$.

Remark 2.1. Let $T$ be a bounded operator on $X$. The following wellknown properties will be often used in the sequel :

(i) Let $z \in X$ and $f \in X^{\prime}$. If $T$ is invertible then it follows from [9, Lemma 4] that

$$
T-z \otimes f \text { is non-invertible if and only if } f\left(T^{-1} z\right)=1 \text {. }
$$

(ii) For every finite rank operator $F \in \mathcal{B}(X)$, we have

$$
T \text { is algebraic if and only if } T+F \text { is algebraic. }
$$

(iii) $T$ is algebraic if and only if $T^{\prime}$ is algebraic.

(iv) For every invertible bounded linear, or conjugate linear, operator $A$ on $X$,

$T$ is algebraic if and only if $A T A^{-1}$ is algebraic. 
(v) If $T$ is algebraic then $\sigma(T)$ is finite and formed by the poles of the resolvent of $T$ of finite order, see [18].

The following proposition describes the adjacency of operators in terms of singular algebraic operators.

Proposition 2.2. Let $A, B \in \mathcal{A}_{0}(X)$. Then the following assertions are equivalent :

(i) $A-B$ is a rank one operator;

(ii) there exists $R \in \mathcal{A}_{0}(X) \backslash\{A, B\}$ such that $R-B \in \mathcal{A}_{s}(X)$ and for every $T \in \mathcal{A}_{0}(X)$, the relations $T-R \in \mathcal{A}_{s}(X)$ and $T-B \in \mathcal{A}_{s}(X)$ yield that $T-A \in \mathcal{A}_{s}(X)$.

We need first to establish the following lemma which will help us to reduce the proof of this proposition to the case of finite-dimensional spaces.

Lemma 2.3. Let $Y$ be a finite-dimensional subspace, and let $Z$ be a closed subspace such that $X=Y \oplus Z$. Let $T \in \mathcal{B}(X)$ be the operator represented by the matrix

$$
T=\left(\begin{array}{cc}
A & B \\
D & C
\end{array}\right)
$$

Then $T$ is algebraic if and only if $C$ is algebraic. Furthermore, if $D=0$ or $B=0$, then $\sigma(T)=\sigma(A) \cup \sigma(C)$.

Proof. Note that since the subspace $Y$ is finite-dimensional, the operators $A, B$ and $D$ are finite-rank. Furthermore, we have

$$
T=\left(\begin{array}{cc}
0 & 0 \\
0 & C
\end{array}\right)+\left(\begin{array}{cc}
A & B \\
D & 0
\end{array}\right)
$$

Hence, it follows from (2.2) that $T$ is algebraic if and only if $C$ is algebraic.

Finally, if $D=0$ or $B=0$, it follows immediately from [4, Corollary 8] that $\sigma(T)=\sigma(A) \cup \sigma(C)$, which completes the proof. 
Proof. [Proof of Proposition 2.2] Note that none of the conditions of Proposition 2.2 is effected if we replace $A$ and $B$ by $A-C$ and $B-C$, respectively, where $C \in \mathcal{A}_{0}(X)$ is any bounded linear operator. Then, without loss of generality we can assume that $B=0$.

(i) $\Rightarrow$ (ii). Suppose that $A$ is a rank one operator. Let $A=z \otimes f$ where $z \in X$ and $f \in X^{\prime}$ are non-zero, and let $R=-A$. Clearly, we have $R \in \mathcal{A}_{s}(X) \backslash\{A, 0\}$. Let $T \in \mathcal{A}_{s}(X)$ be such that $T-R \in \mathcal{A}_{s}(X)$. Since $T$ is algebraic then so is $T-A$, and hence it suffices to show that $T-A$ is non-invertible. Clearly, if $\mathbf{K} e r(T) \cap \mathbf{K} e r(f) \neq\{0\}$ then $\mathbf{K} e r(T-A)$ is non-trivial, and so $T-A \in \mathcal{A}_{s}(X)$. Assume that $\mathbf{K} \operatorname{er}(T) \cap \mathbf{K} \operatorname{er}(f)=\{0\}$. Since $T-R=T+A \in \mathcal{A}_{s}(X)$, it follows that $T+A$ is not injective. Let $u \in \mathbf{K e r}(T+A)$ be non-zero. Then $T u=-f(u) z$, and hence $f(u) \neq 0$, since otherwise $u \in \mathbf{K} e r(T) \cap \mathbf{K} e r(f)$, a contradiction. Let $v=-f(u)^{-1} u$. Then

$$
f(v)=-1, \quad z=T v \text { and } I-v \otimes f \text { is invertible. }
$$

Consequently, $T-A$ is non-invertible because $T-A=T(I-v \otimes f)$ and $T$ is non-invertible.

(ii) $\Rightarrow$ (i). Suppose that $\operatorname{dim} \operatorname{ran}(A) \geq 2$, and let us prove that for every operator $R \in \mathcal{A}_{s}(X) \backslash\{A, 0\}$, there exists $T \in \mathcal{A}_{s}(X)$ such that $T-R \in \mathcal{A}_{s}(X)$ and $T-A \notin \mathcal{A}_{s}(X)$.

So, let $R \in \mathcal{A}_{s}(X) \backslash\{A, 0\}$. Clearly, if $A \notin \mathcal{A}_{s}(X)$ then it suffices to take $T=0$. Assume that $A \in \mathcal{A}_{s}(X)$. In particular, $A$ is not injective. As $R \neq A$, there is a vector $x \in X$ such that $(R-A) x \neq 0$. Since $\operatorname{dim} \operatorname{ran}(A) \geq$ 2 , there exists $y \in X$ such that $(R-A) x, A y$ are linearly independent vectors. Perturbing $y$ by a vector of $\operatorname{Ker}(A)$, we may assume that the vectors $\{x, y\}$ are linearly independent. Let $Z$ be a closed subspace such that $X=\operatorname{Span}\{x, y,(R-A) x, A y\} \oplus Z$. Relatively to this decomposition, the operators $A$ and $R$ can be expressed as follows

$$
A=\left(\begin{array}{cc}
A_{1} & A_{2} \\
A_{3} & A_{4}
\end{array}\right) \text { and } R=\left(\begin{array}{cc}
R_{1} & R_{2} \\
R_{3} & R_{4}
\end{array}\right) .
$$

Consider also the operator $T \in \mathcal{B}(X)$ represented by the matrix

$$
T=\left(\begin{array}{cc}
S+A_{1} & 0 \\
A_{3} & c I
\end{array}\right)
$$

where $c$ is a scalar such that $c I-A_{4}$ is invertible, and $S$ is an invertible operator such that $S x=(R-A) x$ and $S y=-A y$. Clearly, it follows from the previous lemma that $R_{4}$ and $T$, and so $T-R$, are algebraic operators. 
But, since $T y=(T-R) x=0$, we get that $T$ and $T-R$ belong to $\mathcal{A}_{s}(X)$. On the other hand, we have

$$
T-A=\left(\begin{array}{cc}
S & -A_{2} \\
0 & c I-A_{4}
\end{array}\right),
$$

and hence $T-A$ is invertible by Lemma 2.3. Thus, $T-A \notin \mathcal{A}_{s}(X)$. This completes the proof.

We continue with the following proposition.

Proposition 2.4. Let $T, S \in \mathcal{A}_{0}(X)$ be such that

$$
T-N \in \mathcal{A}_{s}(X) \Leftrightarrow S-N \in \mathcal{A}_{s}(X),
$$

for every $N \in \mathcal{A}_{0}(X)$. Then $T=S$.

Proof. Note that for $N=S$ we obtain that $T-S \in \mathcal{A}_{s}(X)$. Hence, in order to prove that $T=S$ it suffices to show that $T-S=c I$ for some scalar $c$. Suppose to the contrary that $T-S$ is not a scalar multiple of the identity. Then, there exists $x \in X$ such that the vectors $x,(T-S) x$ are linearly independent. Let $X=\operatorname{Span}\{x,(T-S) x\} \oplus Z$ where $Z$ is a closed subspace. With respect to this decomposition, we can represent $T-S$ by the matrix

$$
T-S=\left(\begin{array}{cc}
A & B \\
D & C
\end{array}\right) \text { where } A=\left(\begin{array}{cc}
0 & \alpha \\
1 & \beta
\end{array}\right) .
$$

Consider also the operator $R \in \mathcal{B}(X)$ given by

$$
R=\left(\begin{array}{cc}
F & 0 \\
-D & I-C
\end{array}\right) \text { where } F=\left(\begin{array}{cc}
0 & 1-\alpha \\
0 & 0
\end{array}\right)
$$

Note that $R$ is non-invertible because $R x=0$. Since $T-S \in \mathcal{A}_{s}(X)$, Lemma 2.3 asserts that $C$ is algebraic, and so is $R$. Hence, if we let $N=$ $S-R$ we obtain that $S-N=R \in \mathcal{A}_{s}(X)$. But, since

$$
T-N=\left(\begin{array}{cc}
A+F & B \\
0 & I
\end{array}\right) \text { and } A+F=\left(\begin{array}{cc}
0 & 1 \\
1 & \beta
\end{array}\right)
$$

we obtain by Lemma 2.3 that $T-N$ is invertible. This contradiction finishes the proof. 


\section{Proof of the main result}

Combining Proposition 2.4 with Proposition 2.2, we obtain :

Corollary 3.1. Let $\Phi: \mathcal{A}_{0}(X) \rightarrow \mathcal{A}_{0}(X)$ be a surjective map preserving the difference of $\mathcal{A}_{s}(X)$ in both directions. Then

(i) $\Phi$ is injective;

(ii) $\Phi$ preserves adjacency in both directions.

Proof. (i) Let $T, S \in \mathcal{A}_{0}(X)$ be such that $\Phi(T)=\Phi(S)$. For every $N \in \mathcal{A}_{0}(X)$ we have

$T-N \in \mathcal{A}_{s}(X) \Leftrightarrow \Phi(T)-\Phi(N)=\Phi(S)-\Phi(N) \in \mathcal{A}_{s}(X) \Leftrightarrow S-N \in \mathcal{A}_{s}(X)$.

Hence, we get by Proposition 2.4 that $T=S$. Thus, $\Phi$ is injective.

(ii) Let $A, B \in \mathcal{A}_{0}(X)$ be such that $A-B$ has rank one. Then it follows by Proposition 2.2 that there exists $R \in \mathcal{A}_{0}(X) \backslash\{A, B\}$ such that $R-B \in \mathcal{A}_{s}(X)$ and for every $T \in \mathcal{A}_{0}(X)$, the relations $T-R \in \mathcal{A}_{s}(X)$ and $T-B \in \mathcal{A}_{s}(X)$ yield that $T-A \in \mathcal{A}_{s}(X)$. Then $\Phi(R) \in \mathcal{A}_{0}(X) \backslash$ $\{\Phi(A), \Phi(B)\}$ because $\Phi$ is injective, and $\Phi(R)-\Phi(B) \in \mathcal{A}_{s}(X)$. Let $S \in \mathcal{A}_{0}(X)$ be such that $S-\Phi(R) \in \mathcal{A}_{s}(X)$ and $S-\Phi(B) \in \mathcal{A}_{s}(X)$. Since $\Phi$ is surjective, there is $T \in \mathcal{A}_{0}(X)$ such that $S=\Phi(T)$. Thus, $T-R \in \mathcal{A}_{s}(X)$ and $T-B \in \mathcal{A}_{s}(X)$, which yield that $T-A \in \mathcal{A}_{s}(X)$, and so $S-\Phi(A) \in \mathcal{A}_{s}(X)$. Therefore, we obtain by Proposition 2.2 that $\Phi(A)-\Phi(B)$ has rank one. On the other hand, since $\Phi$ is bijective and $\Phi^{-1}$ satisfies the same properties as $\Phi$, we obtain that $\Phi$ preserves adjacency in both directions.

Let $\tau$ be a field automorphism of $\mathbf{K}$. An additive map $A: X \rightarrow Y$ defined between two Banach spaces will be called $\tau$-semi linear if $A(\lambda x)=$ $\tau(\lambda) A x$ holds for all $x \in X$ and $\lambda \in \mathbf{K}$. If $\tau$ is the complex conjugation, we will say simply that $A$ is conjugate linear. Notice that if $A$ is non-zero and bounded, then $\tau$ is continuous, and consequently, $\tau$ is either the identity or the complex conjugation, see Theorem 14.4.2 and Lemma 14.5.1 in [10]. Moreover, in this case, the adjoint operator $A^{\prime}: Y^{\prime} \rightarrow X^{\prime}$ defined by

$$
A^{\prime}(g)=\tau^{-1} \circ g \circ A \text { for all } g \in Y^{\prime},
$$

is again $\tau$-semi linear. 
Lemma 3.2. Let $\Phi: \mathcal{A}_{0}(X) \rightarrow \mathcal{A}_{0}(X)$ be a surjective map preserving the difference of $\mathcal{A}_{s}(X)$ in both directions and such that $\Phi(I)=I+\Phi(0)$. Then either

(i) there exists an invertible bounded linear, or conjugate linear, operator $A: X \rightarrow X$ such that $\Phi(F)=A F A^{-1}+\Phi(0)$ for all finite rank operator $F \in \mathcal{A}_{0}(X)$, or

(ii) there exists an invertible bounded linear, or conjugate linear, operator $B: X^{\prime} \rightarrow X$ such that $\Phi(F)=B F^{\prime} B^{-1}+\Phi(0)$ for all finite rank operator $F \in \mathcal{A}_{0}(X)$. In this case, $X$ is reflexive.

Proof. After replacing $\Phi$ by $T \mapsto \Phi(T)-\Phi(0)$, we may assume that $\Phi(0)=0$. Then, in particular, we have $\Phi(I)=I$. Hence, Corollary 3.1 ensures that $\Phi$ is bijective and preserves adjacency in both directions. Since every rank one operator $F_{1}$ is adjacent to zero and $\Phi(0)=0$, then $\Phi\left(F_{1}\right)$ is a rank one operator. Analogously, since every rank two operator $F_{2}$ is adjacent to a rank one operator, then $\Phi\left(F_{2}\right)$ is a finite rank operator. By repeating the same argument we infer that $\Phi$ maps the subspace $\mathcal{F}(X) \subset$ $\mathcal{A}_{0}(X)$ of all finite rank operators onto itself.

Then, by [14, Theorem 1.5], $\Phi_{\mid \mathcal{F}(X)}$ is an additive map and there exist a ring automorphism $\tau: \mathbf{K} \rightarrow \mathbf{K}$ and either two bijective $\tau$-semi linear mappings $A: X \rightarrow X$ and $C: X^{\prime} \rightarrow X^{\prime}$ such that

$$
\Phi(x \otimes f)=A x \otimes C f \quad \text { for all } x \in X \text { and } f \in X^{\prime},
$$

or two bijective $\tau$-semi linear mappings $B: X^{\prime} \rightarrow X$ and $D: X \rightarrow X^{\prime}$ such that

$$
\Phi(x \otimes f)=B f \otimes D x \quad \text { for all } x \in X \text { and } f \in X^{\prime} .
$$

Suppose that $\Phi$ satisfies (3.1), and let us show that

$$
C(f)(A x)=\tau(f(x)) \quad \text { for all } x \in X \text { and } f \in X^{\prime} .
$$

Clearly, it suffices to establish that for all $x \in X$ and $f \in X^{\prime}, f(x)=1$ if and only if $C(f)(A x)=1$. Let $x \in X$ and $f \in X^{\prime}$. Then

$$
f(x)=1 \Leftrightarrow I-x \otimes f \in \mathcal{A}_{s}(X) \Leftrightarrow I-A x \otimes C f \in \mathcal{A}_{s}(X) \Leftrightarrow C(f)(A x)=1 .
$$


Thus, equation (3.3) holds, and arguing as in [12, p. 252], we get that $\tau, A, C$ are continuous, $\tau$ is the identity or the complex conjugation, and $C=\left(A^{-1}\right)^{\prime}$. Therefore, $\tau^{-1}=\tau$ and, for every $u \in X$, we have

$$
\Phi(x \otimes f) u=\tau\left(f A^{-1} u\right) A x=A\left(f\left(A^{-1} u\right) x\right)=A(x \otimes f) A^{-1} u .
$$

Thus, $\Phi(x \otimes f)=A(x \otimes f) A^{-1}$ for all $x \in X$ and $f \in X^{\prime}$. Hence, $\Phi(F)=A F A^{-1}$ for all finite rank operator $F$.

Now, suppose that $\Phi$ satisfies (3.2). In a similar way as (3.1), we establish that

$$
D(x)(B f)=\tau(f(x)) \quad \text { for all } x \in X \text { and } f \in X^{\prime} .
$$

Moreover, arguing as in [12, p. 252], we get that $\tau, B, D$ are continuous, $\tau$ is the identity or the complex conjugation, and $D=\left(B^{-1}\right)^{\prime} \mathrm{J}$, where $\mathrm{J}: X \rightarrow X^{\prime \prime}$ is the natural embedding. But, since $D$ and $\left(B^{-1}\right)^{\prime}$ are bijective, $\mathrm{J}$ is bijective and $X$ is reflexive. Furthermore, $\tau^{-1}=\tau$ and, for every $u \in X$, we have

$$
\begin{aligned}
\Phi(x \otimes f) u & =\left(B f \otimes\left(B^{-1}\right)^{\prime} \mathrm{J}(x)\right) u=\left(B^{-1}\right)^{\prime} \mathrm{J}(x)(u) \cdot B f \\
& =\tau\left(\mathrm{J}(x)\left(B^{-1} u\right)\right) \cdot B f=B\left(\mathrm{~J}(x)\left(B^{-1} u\right) f\right) \\
& =B(f \otimes \mathrm{J}(x)) B^{-1} u=B(x \otimes f)^{\prime} B^{-1} u .
\end{aligned}
$$

Thus, $\Phi(x \otimes f)=B(x \otimes f)^{\prime} B^{-1}$ for all $x \in X$ and $f \in X^{\prime}$. Hence, $\Phi(F)=B F^{\prime} B^{-1}$ for all finite rank operator $F$. This completes the proof.

Let $\mathcal{A}_{i}(X)$ denote the set of all invertible algebraic operators in $\mathcal{B}(X)$.

Lemma 3.3. Let $T, S \in \mathcal{A}_{s}(X)$ be such that

$$
T-N \in \mathcal{A}_{i}(X) \Leftrightarrow S-N \in \mathcal{A}_{i}(X),
$$

for every $N \in \mathcal{A}_{i}(X)$. Then $T=S$.

Proof. Note that $T-\alpha I$ is invertible if and only if $S-\alpha I$ is invertible for every non-zero scalar $\alpha$. This implies that $\sigma(T)=\sigma(S)$. Consequently, it suffices to establish that $T=S+\lambda I$ for some scalar $\lambda$, because in this case $\sigma(S)=\sigma(S+\lambda)$, and so $\lambda=0$. 
Suppose to the contrary that there exists $x \in X$ such that $\{x,(T-S) x\}$ is a linearly independent set. It follows that either $\{x, T x\}$ or $\{x, S x\}$ is linearly independent. There is no loss of generality in assuming that $\{x, T x\}$ is linearly independent. Let $Y=\operatorname{Span}\{x, T x, S x\}$, and let $Z$ be a closed subspace such that $X=Y \oplus Z$. With respect to this decomposition, the operator $S$ can be written as follows

$$
S=\left(\begin{array}{ll}
S_{1} & S_{2} \\
S_{3} & S_{4}
\end{array}\right)
$$

Note that if $S x=\alpha x+\beta T x$ for some scalars $\alpha$ and $\beta$, then $\beta \neq 1$ because $\{x,(T-S) x\}$ is a linearly independent set. Thus, one can easily find an invertible linear operator $A: Y \rightarrow Y$ such that $A x=T x$ and $S_{1}-A$ is invertible. Consider the operator $N \in \mathcal{B}(X)$ given by the matrix

$$
N=\left(\begin{array}{cc}
A & S_{2} \\
0 & c I
\end{array}\right)
$$

where $c$ is a non-zero scalar such that $S_{4}-c I$ is invertible. Since $A$ and $S_{1}-A$ are invertible, Lemma 2.3 ensures that $N$ and $S-N$ are invertible algebraic operators. But, since $(T-N) x=0$, we obtain that $T-N \notin \mathcal{A}_{i}(X)$, a contradiction.

Lemma 3.4. Let $\Phi: \mathcal{A}_{0}(X) \rightarrow \mathcal{A}_{0}(X)$ be a surjective map preserving the difference of $\mathcal{A}_{s}(X)$ in both directions and such that $\Phi(I)=I$ and $\Phi(F)=F$ for all finite rank operator $F$. Then $\Phi(T)=T$ for all $T \in \mathcal{A}_{0}(X)$.

Proof. Corollary 3.1 implies that $\Phi$ is bijective and preserves adjacency in both directions. We shall divide the proof into four steps.

Step 1. $\Phi$ preserves the difference of $\mathcal{A}_{i}(X)$ in both directions. Let $T, S \in \mathcal{A}_{0}(X)$ be such that $T-S \in \mathcal{A}_{i}(X)$. Then there exist $z \in X$ and $f \in X^{\prime}$ such that $f\left((T-S)^{-1} z\right)=1$. Put $F=z \otimes f$. Since $T-(S+F)$ is algebraic and non-invertible by $(2.1)$, we have $T-(S+F) \in \mathcal{A}_{s}(X)$. Thus, $\Phi(T)-\Phi(S+F) \in \mathcal{A}_{s}(X)$. On the other hand, since $(S+F)-S$ is rank one then so is $\Phi(S+F)-\Phi(S)$, and consequently

$$
\Phi(T)-\Phi(S)=\Phi(T)-\Phi(S+F)+\Phi(S+F)-\Phi(S)
$$


is an algebraic operator. But, since $T-S \notin \mathcal{A}_{s}(X)$, we have $\Phi(T)-\Phi(S) \notin$ $\mathcal{A}_{s}(X)$, and hence $\Phi(T)-\Phi(S) \in \mathcal{A}_{i}(X)$. Since $\Phi^{-1}$ satisfies the same properties as $\Phi$, we obtain that $\Phi$ preserves the difference of $\mathcal{A}_{i}(X)$ in both directions.

Step 2. $\Phi(T)=T$ for every operator $T \in \mathcal{A}_{i}(X)$. Let $T \in \mathcal{A}_{i}(X)$. Since $\Phi$ preserves the difference of $\mathcal{A}_{i}(X)$ in both directions and $\Phi(0)=0$, then $\Phi(T) \in \mathcal{A}_{i}(X)$. If $\Phi(T) \neq T$, there exist $x \in X$ and $g \in X^{\prime}$ such that $T^{-1} x \neq \Phi(T)^{-1} x, g\left(T^{-1} x\right)=1$ and $g\left(\Phi(T)^{-1} x\right) \neq 1$. Hence, we get by (2.1) that $T-x \otimes g \notin \mathcal{A}_{i}(X)$ and $\Phi(T)-\Phi(x \otimes g)=\Phi(T)-x \otimes g \in \mathcal{A}_{i}(X)$. This contradiction shows that $\Phi(T)=T$.

Step 3. $\Phi(T)=T$ for every operator $T \in \mathcal{A}_{s}(X)$. Let $T \in \mathcal{A}_{s}(X)$. Clearly, $\Phi(T) \in \mathcal{A}_{s}(X)$. For every $N \in \mathcal{A}_{i}(X)$, we have $\Phi(N)=N$ and

$$
T-N \in \mathcal{A}_{i}(X) \Leftrightarrow \Phi(T)-N \in \mathcal{A}_{i}(X) .
$$

Thus, we get by Lemma 3.3 that $\Phi(T)=T$.

Step 4. Now, we prove the statement inductively. Without loss of generality, it suffices to establish that $\Phi\left(T_{1}+T_{2}\right)=T_{1}+T_{2}$ for every algebraic operators $T_{1}, T_{2} \in \mathcal{B}(X)$. Let $T_{1}, T_{2} \in \mathcal{B}(X)$ be algebraic, and consider the following map

$$
\Psi(T)=\Phi\left(T+T_{2}\right)-T_{2} \quad \text { for all } T \in \mathcal{A}_{0}(X) .
$$

Clearly, $\Psi$ is bijective and preserves the difference of $\mathcal{A}_{s}(X)$ in both directions. Furthermore, it follows from the previous steps that $\Psi(I)=I$ and $\Psi(F)=F$ for all finite rank operator $F$. Hence, we get that $\Psi(T)=T$ for every algebraic operator $T \in \mathcal{B}(X)$. In particular, we get that $\Psi\left(T_{1}\right)=$ $\Phi\left(T_{1}+T_{2}\right)-T_{2}=T_{1}$. This shows that $\Phi\left(T_{1}+T_{2}\right)=T_{1}+T_{2}$, as desired.

With these results at hand, we are ready to prove our main theorem.

Proof. $\quad[$ Proof of Theorem A] (i) $\Rightarrow$ (ii). Suppose that $\Phi$ preserves the difference of $\mathcal{A}_{s}(X)$ in both directions. It follows that $\Phi$ takes one of the two forms in Lemma 3.2.

Assume that $\Phi(F)=A F A^{-1}+\Phi(0)$ for all finite rank operator $F \in$ $\mathcal{B}(X)$. Let

$$
\Psi(T)=A^{-1}(\Phi(T)-\Phi(0)) A \quad \text { for all } T \in \mathcal{A}_{0}(X) .
$$

Clearly, $\Psi$ satisfies the same properties as $\Phi$. Furthermore, $\Psi(I)=I$ and $\Psi(F)=F$ for all finite rank operator $F \in \mathcal{B}(X)$. Hence, we get by 
the previous lemma that $\Psi(T)=T$ for all $T \in \mathcal{A}_{0}(X)$. This shows that $\Phi(T)=A T A^{-1}+\Phi(0)$ for all $T \in \mathcal{A}_{0}(X)$.

Now suppose that $\Phi(F)=B F^{\prime} B^{-1}+\Phi(0)$ for all finite rank operator $F \in \mathcal{B}(X)$. Then, Lemma 3.2 ensures that $X$ is reflexive. By considering

$$
\Gamma(T)=\mathrm{J}^{-1}\left(B^{-1}(\Phi(T)-\Phi(0)) B\right)^{\prime} \mathrm{J} \quad \text { for all } T \in \mathcal{A}_{0}(X),
$$

we get in a similar way that $\Gamma(T)=T$ for all $T \in \mathcal{A}_{0}(X)$. Thus, $\Phi(T)=B T^{\prime} B^{-1}+\Phi(0)$ for all $T \in \mathcal{A}_{0}(X)$, as desired.

(ii) $\Rightarrow$ (i) is obvious.

We close this paper by the following remark and question :

Remark 3.5. Let $Y$ be an infinite-dimensional Banach space over $\mathbf{K}$. Theorem $A$ can be formulated without any change for surjective maps $\Phi: \mathcal{A}_{0}(X) \rightarrow \mathcal{A}_{0}(Y)$, with $\Phi(I)=I+\Phi(0)$, preserving the difference of singular algebraic operators in both directions.

Question 3.6. Is Theorem A true without supposing $\Phi(I)=I+\Phi(0)$ ?

\section{References}

[1] Z. F. BAI AND J. Hou, Linear maps and additive maps that preserve operators annihilated by a polynomial, J. Math. Anal. Appl. 271, pp. 139-154, (2002).

[2] Z. F. BAI AND J. Hou , Additive maps preserving nilpotent operators or spectral radius, Acta Math. Sin. (Engl. Ser.) 21, pp. 1167-1182, (2005).

[3] A. Bourhim, J. Mashreghi and A. Stepanyan, Nonlinear maps preserving the minimum and surjectivity moduli, Linear Algebra Appl. 463, pp. 171-189, (2014).

[4] J. K. Han, H. Y. Lee And W. Y. LeE, Invertible completions of $2 \times 2$ upper triangular operator matrices, Proc. Amer. Math. Soc. 128, pp. 119-123, (2000).

[5] H. HavliceK ANd P. Šemrl, From geometry to invertibility preservers, Studia Math. 174, pp. 99-109, (2006). 
[6] J. Hou And L. Huang, Characterizing isomorphisms in terms of completely preserving invertibility or spectrum, J. Math. Anal. Appl. 359, pp. 81-87, (2009).

[7] L. K. HuA, A theorem on matrices over a field and its applications, Acta Math. Sin. (Engl. Ser.) 1, pp. 109-163, (1951).

[8] W.-L. Huang And P. Šemrl, Adjacency preserving maps on Hermitian matrices, Canad. J. Math. 60, pp. 1050-1066, (2008).

[9] A. A. Jafarian And A. R. Sourour, Spectrum-preserving linear maps, J. Funct. Anal. 66, pp. 255-261, (1986).

[10] M. Kuczma, An Introduction to the Theory of Functional Equations and Inequalities, Państwowe Wydawnictwo Naukowe, Warszawa, (1985).

[11] V. MüLLER, Spectral Theory of Linear Operators and Spectral Systems in Banach Algebras. Second edition. Operator Theory: Advances and Applications, 139. Birkhäuser Verlag, Basel, (2007).

[12] M. Omladič AND P. ŠEmRL, Additive mappings preserving operators of rank one, Linear Algebra Appl. 182, pp. 239-256, (1993).

[13] C. Pearcy and D. Topping, Sums of small numbers of idempotents, Michigan Math. J. 14, pp. 453-465, (1967).

[14] T. Petek And P. Šemrl, Adjacency preserving maps on matrices and operators, Proc. Roy. Soc. Edinburgh 132A, 661-684, (2002).

[15] P. ŠemrL, On Hua's fundamental theorem of the geometry of rectangular matrices, J. Algebra 248, pp. 366-380, (2002).

[16] P. ŠEmRL, Hua's fundamental theorem of the geometry of matrices, J. Algebra 272, pp. 801-837, (2004).

[17] K. Soullah, On additive preservers of certain classes of algebraic operators, Extracta Math. 30, pp. 207-220, (2015).

[18] A. E. TAYlor And D. C. LAY, Introduction to Functional Analysis, Wiley, New York-Chichester-Brisbane, (1980).

[19] Z. Wan, Geometry of Matrices, World Scientific Publishing Co., Singapore, (1996). 


\section{Mourad Oudghiri}

Département Math-Info,

Labo LAGA,

Faculté des Sciences d'Oujda,

Université Mohammed Premier - Oujda, 60000 Oujda,

Maroc

e-mail : morad.oudghiri@gmail.com

and

\section{Khalid Souilah}

Département Math-Info, Labo LAGA,

Faculté des Sciences d'Oujda, 60000 Oujda,

Maroc

e-mail : s.khalide@gmail.com 